\title{
Communication and technology
}

\section{Charles M. Ess}

To cite this article: Charles M. Ess (2017): Communication and technology, Annals of the International Communication Association

To link to this article: http://dx.doi.org/10.1080/23808985.2017.1392250

Published online: 30 Oct 2017.

I introduce this double issue, showing first how the articles conjoin global perspectives with fine-grained attention to specific communication venues vis-à-vis critical theory, politics, religion, children and youth, mobile technologies, and ICT4D. Our contributors further argue against technological determinism in multiple ways, offering instead far more sophisticated understandings of how technology, material factors, and diverse ethical, social, political and cultural - i.e., human - factors interact. Finally, the normative dimensions and trajectories of the contributions counter positivist notions of technological instrumentalism (technology as "value free") and likewise foreground multiple ethical foundations and ethically-informed approaches. These accounts thus provide both focused explorations of contemporary research findings and trajectories in specific domains while also tracing out larger thematics in communication and technology.

\section{Introduction: contra ethnocentrism}

To begin with the obvious, "communication and technology" conjoin enormously broad and complex territories. A prime challenge follows from the initial recognition that both 'communication' and 'technology' are core constituents of human beings and their manifold familial, social, and political collocations in every time and place (e.g., Peters 1999, 2015). At the same time, as we have learned from more than two decades of Internet Studies (e.g., Consalvo and Ess 2011; cf. Nocera et al 2016): as communication technologies both diffuse throughout the whole of our lives in the (so-called) developed world and connect and bind us globally - approaching communication technologies demands both (a) specificity and precision of focus - i.e., close attention to specific instantiations of communication and technologies, and (b) a global scope, one that encompasses the often staggering cultural, 
ethical, social, and political diversities - diversities made all the more apparent precisely by these technologies - so as to avoid ethnocentrism first of all.

To address this complex of issues, we have developed six articles that trace out a conceptual trajectory. Starting with the most general, we take up Maria Bakardjieva and Robert W Gehl's overview of the diverse forms of critical theoretical approaches to communication technology. The next three authors then focus on a specific dimension of technologically mediated communication, beginning with Gunn Enli's examination of politics and communication. Heidi Campbell explores religion and communication, followed by Elizabeth Staksrud and Tijana Milesovec's attention to children and youth. We then turn to Rich Ling's analyses of mobile technologies as these have dramatically transformed our communicative practices and possibilities. We conclude with José Abdelnour-Nocera and Melissa Densmore's overview of one of the most compelling domains for the development and application of communication technologies, namely ICT4D - ICTs for development.

Given the size limits of each issue of the Annals, however, we have had to distribute these articles across two issues: the first three (Bakardjieva \& Gehl, Enli, Campbell) are presented here - the second three (Staksrud \& Milosevec, Ling, and Abdelnour-Nocera \& Densmore) will appear in the following issue (Vol. 1, issue 4, December 2017).

Each of these contributions offers an overview of some of the most significant of the primary literatures, research questions and findings, and promising directions for future research. Moreover, each foregrounds important shifts from earlier, more U.S.- or Westerncentric research and perspectives towards more encompassing and global ones. To begin with, critical theories originated in the Western Enlightenment and conceptions of liberaldemocratic polities as required and legitimated by the classical liberal subject: while a good portion of these remain central to critical theoretical concerns in the contemporary world, they 
are dramatically challenged and modified, e.g., by various feminist critiques as well as by one of the most significant threats to democratic norms and processes - namely, the platforms and algorithms that constitute our primary communicative environments, but as thereby the property and business enterprises of a handful of multinational corporations (Bakardjieva and Gehl). Similarly, Enli's account of the political dimensions of communication technology highlights the failures of these technologies to "democratize" in many parts of the world, most famously in the example of the Arab Spring. Staksrud and Milosevec's attention to children and youth includes both the EU Kids Online and the more recent Global Kids Online project. Religion as manifestly a global phenomenon hence requires global attention and research (Campbell). At the same time, mobile technologies have diffused in dramatic ways in both developed and developing countries (Ling). Last, but certainly not least, ICT4D (AbdelnourNocera and Densmore) most explicitly addresses the contrasts, conflicts, and possible resolutions between initial, primarily Western assumptions, aims, and hopes for ICTs in these contexts, on the one hand, and, on the other, the local cultural and social traditions and realities of specific peoples in the developing world.

\section{Contra technological determinism}

Moreover, both individually and collectively, these accounts offer important, empiricallygrounded insights into larger questions and issues that cluster about technology and communication. One of the oldest of these, at least since the foundation of media effects studies, is precisely the concern - or outright fear - that new communication technologies threaten both individuals, beginning with children and youth, and larger institutions, including the institutions and norms of democratic polity. Perhaps the strongest expression of these concerns is the view of technological determinism. Once characterized in terms of "autonomous technology" (Winner 1978), this view assumes that once technological genies are released from their bottles, they will evoke their impacts, for better and for worse, outside 
the possibility of human steering or control. This view, in fact, is rooted in the Romantic reaction against the emergence of modern rationalism, science, and technologies (Ess 2017b). It predominated especially in 1990s' hopes that "wiring the world" with the internet would inevitably result in democracy, freedom, and prosperity. In a number of ways, this view was roundly refuted by the end of the 1990s (Ess and Consalvo 2011). Here, Enli reiterates the point: contra the ostensible democratization promise of new communication technologies, politicians (among others) have proven adept at short-circuiting and rerouting these technologies in ways that sustain and enhance their power and authority, rather than facilitating challenge, debate, and institutional transformation. Campbell makes the same point with regard to religious institutions and authorities. Perhaps most dramatically, Abdelnour-Nocera and Densmore document how "Western" communication technologies designed precisely to foster and enhance democratic norms of transparency, equality, and so on, thereby directly clash with local social and political structures that may include, for example, great respect for an elder's opinion or view as a deeply rooted cultural norm. Of course, none of this is to say that new technologies cannot be transformative in positive ways: but these transformations precisely entail an array of additional human (social, ethical, political ...) and material factors. So Rich Ling explores the specific social processes required for developing a critical mass of users of a specific communication app, resulting in the app then becoming the "app de l'emplacement" (the app required for communication in a specific location, such as a city, region, or country). Once in place, such apps afford new communicative practices, including what Ling calls "indirect event coordination." By the same token, Campbell details how the contemporary weaving of communication technologies into everyday life makes possible new forms of religion and spirituality. Staksrud and Milosevec likewise highlight an important shift from initial concerns to protect children and youth from ostensible dangers of new technologies towards the more contemporary emphasis 
on "the right to participate as active agents in spaces that are of crucial importance for their cognitive and emotional development, while being provided with media content and technological affordances that foster this development."

\section{Normative dimensions}

As this last comment exemplifies, there is a strong thread of shared normative interests, claims and arguments running throughout our contributions. From especially more contemporary perspectives, this is inevitable: a host of developments - both within the natural sciences (such a relativity theories and then Quantum Mechanics) and then in numerous critiques of positivism emerging in the $2^{\text {nd }}$ half of the $20^{\text {th }}$ century have rather thoroughly undermined positivist and positivist-inspired notions of science and technology as somehow value free or morally neutral. As I have argued elsewhere, once we recognize that value neutrality, however useful as a heuristic in scientific disciplines, is in practice impossible - it then becomes incumbent upon us to articulate and defend our ethical norms, principles, and practices as explicitly as possible (Ess, 2017a).

Here, Abdelnour-Nocera and Densmore make these points as they foreground the conflict between "the assumptions, priorities and values embedded in the tools and concepts" and the norms, practices, interests, and so on defining local cultures. They go on to highlight new ways in which HCI can thus meet the normative demands to be responsible and accountable within specific cultural contexts. More broadly, normative commitments and foundations are apparent from the outset in Bakardjieva and Gehl, as they point out that "the "critical tradition" in communication studies is characterized by a normative orientation anchored in liberal-democratic ethical and political values." Key normative values here are "social justice, equality, emancipation and democratic participation," along with the awareness that communication technologies can also amplify power imbalances and "generate new forms of exploitation and domination." They further point to both feminist and virtue 
ethics sources for extending the normative interests initially rooted in Marxian political economy. By the same token, Enli's analyses begins with identifying the "idealistic functions, such as informed democracy, deliberative debate, and empowerment of the citizens," as well as "feared effects such as polarization, echo chambers, propaganda, miscommunication and "fake news"" that stake out the positives and negatives defined by normative commitments to deliberative democracy and its norms, processes, and so on.

In these directions, Staksrud and Milosevec take up the more specific normative commitments to protecting children while also, as we have seen, endorsing their rights to participation. In addition, they point out a critical danger in the prevailing concerns to protect children - namely, that these good intentions are often exploited for the sake of restricting freedom of expression, and thereby diluting a key right and practice of deliberative democracy. As a final example, in parallel with Abdelnour-Nocera and Densmore, Campbell points to a number of contemporary theories that take on board the value-laden aspects of technology, including her own "religious-social shaping of technology," inspired in part by SCOT (social construction of technology) theories. At the same time, Campbell highlights the recent resurgence of existential questions at play in both our uses of digital media and thereby in our research thereupon - e.g., the themes of "death, time, being there, and being-inand-with-the-world" as brought forward in the work of Amanda Lagerkvist (2017).

These developments parallel similar expansions in attention to ethics, including virtue ethics, in related domains, beginning with ICT design (Spiekermann 2016), networked systems design (Zevenbergen et al, 2015), and autonomous systems design (IEEE). In addition, as social robots - including carebots and sexbots - continue their rapid development and deployment, the ethical, social, political and related normative aspects of these devices as increasingly common embodiments of communication and technology will become ever more urgent and compelling (Ess, 2016). 


\section{Conclusion}

To be sure, there are many, many more topics and domains that would need to be included in a more comprehensive account - beginning with the intersections between technology and interpersonal communication, organizational communication, and new media and culture as a start (Weiyu Zhang, pers. com.). At the same time, we believe that these contributions both individually and collectively offer the requisite fine-grained accounts of contemporary and future directions of findings and research in specific and core domains of communication and technology: I further hope that the broader connections with the larger literatures sketched out above at least begin to argue that these accounts further provide crucial snapshots that also trace out larger patterns and trajectories in these domains.

\section{References}

Abdelnour-Nocera, J., Strano, M., Van der Velden, M., Hrachovec, H., and Ess, C. (eds.). 2016. Culture, Technology, Communication. Common World, Different Futures. 10th IFIP WG 13.8 International Conference, CaTaC 2016, London, UK, June 15-17, 2016, Revised Selected Papers. Springer.

Consalvo, Mia and Ess, Charles (eds.). 2011. The Handbook of Internet Studies. Oxford: Wiley-Blackwell.

Ess, Charles. 2016. What's love got to do with it? Robots, sexuality, and the arts of being human. In M. Nørskov (ed.), Social Robots: Boundaries, Potential, Challenges, 57-79. Ashgate: Farnham, Surrey, England. 2016.

Ess, Charles. 2017a. Can we say anything ethical about Digital Religion? Philosophical and Methodological considerations. New Media \& Society 19(1): 34-42. DOI: $10.1177 / 1461444816649914$.

Ess, Charles. 2017b. God Out of the Machine?: The Politics and Economics of Technological Development. In A. Beavers (ed.), Macmillan Interdisciplinary Handbooks: Philosophy, 83-111. Farmington Hills, MI: Macmillan Reference.

Ess, Charles and Consalvo, Mia. 2011. What is "Internet Studies"? In Consalvo and Ess (eds.), The Handbook of Internet Studies, 1-8. Oxford: Wiley-Blackwell.

IEEE. n.d. Global Initiative for Ethical Considerations in the Design of Autonomous Systems. $<$ http://standards.ieee.org/develop/indconn/ec/autonomous_systems.html $>$ 
Lagerkvist, A. (2017). Existential media: Toward a theorization of digital thrownness. New Media and Society, 19(1), 96-110.

Peters, John. D. 1999. Speaking into the Air: A History of the Idea of Communication. Chicago, IL: University of Chicago Press.

Peters, John. D. 2015. The Marvelous Clouds: Toward a Philosophy of Elemental Media. Chicago: Chicago University Press.

Spiekermann, Sarah. 2016. Ethical IT Innovation: A Value-based System Design Approach. New York: Taylor \& Francis.

Winner, Langdon. 1978. Autonomous Technology: Technics-out-of-Control as a Theme in Political Thought. Cambridge, MA: MIT Press.

Zevenbergen, Bendert, Mittelstadt, Brent, Véliz, Carissa, Detweiler, Chris, Cath, Corinne, Savulescu, Julian, and Whittaker, Meredith. 2015. Philosophy meets Internet Engineering: Ethics in Networked Systems Research. (GTC workshop outcomes paper). Oxford Internet Institute, University of Oxford. 